\title{
SOME FIXED POINT THEOREMS FOR MULTIVALUED MAPS IN ORDERED BANACH SPACES AND APPLICATIONS
}

\author{
ZHAI CHENGBO AND YANG CHEN
}

Received 10 November 2004 and in revised form 10 July 2005

The existence of maximal and minimal fixed points for various set-valued operators is discussed. This paper presents some new fixed point theorems in ordered Banach spaces. A necessary and sufficient condition for the existence of the fixed point to a class of multivalued maps has been obtained. The uniqueness of the positive fixed point has been discussed. The results extend and improve the corresponding results. As an application, we utilize the results to study the existence and uniqueness of positive fixed points for a class of convex operators. In the end, we give a simple application to certain integral equations.

\section{Introduction}

This paper presents some new fixed point theorems for multivalued maps in ordered Banach spaces. Our arguments rely on the properties of closed convex cone in real Banach spaces (see $[6,8]$ ) and the Kuratowski measure of noncompactness [9]. Our results extend and improve the corresponding results presented in the literature (see [1], [5, Chapter 3]).

We proceed as follows. In Section 2, we present basic concepts and results in ordered Banach spaces. In Section 3, the main results and proofs are given. The results in [1] and [5, Chapter 3] turn out to be special cases of our main results.

Motivated by the ideas and results in [11], we give in Section 4 a necessary and sufficient condition for the existence of the fixed point to a class of multivalued maps which satisfy certain conditions. At the end of Section 4, the uniqueness of the positive fixed point has been discussed sketchily. To demonstrate the applicability of our results, we study the existence and uniqueness of positive fixed points for a class of convex operators and give in the final section of the paper a simple application to certain integral equations.

\section{Preliminaries}

In this section, we summarize some basic concepts and results in real Banach spaces.

Let $X$ be a real Banach space and let $S$ be a nonempty subset of $X$. Suppose that $F$ : $S \rightarrow 2^{X}$; here $2^{X}$ denotes the family of nonempty subsets of $X$. Then $F$ is said to be upper 
semi-continuous at $x_{0} \in S$ if for any $x_{n} \in S,\left\{x_{n}\right\} \rightarrow x_{0}($ as $n \rightarrow \infty)$ and $y_{n} \in F\left(x_{n}\right),\left\{y_{n}\right\} \rightarrow$ $y_{0}($ as $n \rightarrow \infty)$ implies that $y_{0} \in F\left(x_{0}\right)$. If $F$ is upper semicontinuous at each point of $S$, then $F$ is called upper semi-continuous on $S$. Point $x \in S$ is called a fixed point of $F$ if $x \in F(x)$.

If $A$ is a subset of $X$ and $c$ is a real number, we denote $c A=\{c a: a \in A\}$. Let $\Omega_{X}$ be bounded subsets of $X$, the Kuratowski measure of noncompactness is the map $\alpha: \Omega_{X} \rightarrow$ $[0,+\infty)$ defined by (here, $D \in \Omega_{X}$ )

$$
\alpha(D)=\inf \left\{r>0: D \subset \bigcup_{i=1}^{n} D_{i} \text { and } \operatorname{diam}\left(D_{i}\right) \leq r\right\} .
$$

Then we have (see $[10,12,13])$

(1) $\alpha(A)=0 \Leftrightarrow A$ is a relatively compact set;

(2) $A \subset B \Rightarrow \alpha(A) \leq \alpha(B)$, here $A, B$ are bounded subsets of $X$; and

(i) $F: S \rightarrow 2^{X}$ is $k$-set contractive (here $k \geq 0$ ) if $\alpha(F(W)) \leq k \alpha(W)$ for all nonempty, bounded sets $W$ of $S$;

(ii) $F: S \rightarrow 2^{X}$ is condensing if $F$ is 1 -set contractive and $\alpha(F(W))<\alpha(W)$ for all nonempty, bounded sets $W$ of $S$ with $\alpha(W) \neq 0$.

Following the terminology in [10], we say that a nonempty subset $P \subset X$ is a convex cone if $P+P=P, \lambda P \subset P$ for all real number $\lambda \geq 0$. If the cone is pointed, (i.e., $P \cap-P=$ $\{0\})$ such a cone $P$ induces an ordering on $X$. For $x, y \in X$, we write $x \geq y$ if $x-y \in P$. If $x \leq y$, the set $[x, y]=\{z \in X \mid x \leq z \leq y\}$ is called the order interval between $x$ and $y$.

Putting $\stackrel{\circ}{P}=\{x \in P \mid x$ is an interior point of $P\}$, a cone $P$ is said to be solid if its interior $\stackrel{\circ}{P}$ is nonempty. In the case $y-x \in \stackrel{\circ}{P}$, we write $x \ll y$.

For all $x, y \in X$, the notation $x \sim y$ means that there exist $\lambda>0$ and $\mu>0$ such that $\lambda x \leq y \leq \mu x$; clearly, $\sim$ is an equivalence relation. Given $h>\theta$ (i.e., $h \geq \theta$ and $h \neq \theta$ ), we denote by $P_{h}$ the set

$$
P_{h}=\{x \in X \mid \text { there exist } \lambda(x), \mu(x)>0 \text { such that } \lambda(x) h \leq x \leq \mu(x) h\},
$$

and it is easy to see that $P_{h} \subset P$. These concepts discussed above can be found in $[5,6]$.

We will need the following definition $[7,8]$.

Definition 2.1. Given a closed convex cone $P$ in the real Banach space $X$, the following exist.

(i) $P$ is normal if there exists $b>0$, for all $x, y \in P: x \leq y \Rightarrow\|x\| \leq b\|y\|$.

(ii) $P$ is regular if every monotonically decreasing sequence $x_{1} \geq x_{2} \geq \ldots$, which is bounded from below by some element $\bar{x} \in X$, converges in norm.

(iii) $P$ is strongly minimal if it follows from any bounded above set $D \subset X$ that $D$ has supremum.

Definition 2.2 (see [14]). Let $A$ be a positive single-valued mapping on $\stackrel{\circ}{P}$ and let $\alpha \in \mathbb{R}$. Then, say $A$ is $\alpha$-concave ( $\alpha$-convex) if and only if $A(t x) \geq t^{\alpha} A x\left(A(t x) \leq t^{\alpha} A x\right)$ for all $x \in \stackrel{\circ}{P}$ and $t \in(0,1]$.

Let $A$ be a positive mapping on $\stackrel{\circ}{P}$ which is $\alpha$-concave $(\alpha \in[0,1])$, and choose $h \in \stackrel{\circ}{P}$, then $P_{h}=\stackrel{\circ}{P}$. So there exist $\lambda, \mu \in \mathbb{R}^{+}$( $\mathbb{R}^{+}$denotes the positive reals) such that $\lambda h \leq A x \leq \mu h$ 
and $A(t x) \geq t^{\alpha} A x \geq t A x$ for $t \in(0,1]$. Thus $\alpha$-concave mappings are concave in the sense of Martin (see [12, Page 185]). Similar remarks apply to $\alpha$-convex mappings. Note also that $A$ is $\alpha$-concave ( $\alpha$-convex) if and only if $A(s x) \leq s^{\alpha} A x\left(A(s x) \geq s^{\alpha} A x\right)$ for all $x \in \stackrel{\circ}{P}$ and $s \geq 1$.

The following statements play a very important role in the proofs of main results. For $A, B \subset X$, we write $A \leq^{s} B\left(A \geq^{s} B\right)$ if $a \leq b(a \geq b)$ for any $a \in A, b \in B$. A map $F: S \rightarrow 2^{X}$ is said to be increasing if $x \leq y$ implies that $F(x) \leq^{s} F(y)$. As a direct consequence, we have that if $A \leq{ }^{s} A$, then $A$ is a singleton.

\section{Maximal and minimal fixed point theorems}

Throughout this section, we always assume that $X$ is a real Banach space with a partial order introduced by a closed convex cone $P$ of $X$. Take $u_{0}, v_{0} \in X, u_{0}<v_{0}$ and $D=\left[u_{0}, v_{0}\right]$.

Theorem 3.1. Let $F: D \rightarrow 2^{D}$ be an upper semicontinuous, increasing mapping with nonempty value and let $P$ be a regular cone. Then $F$ has maximal and minimal fixed points on $\left[u_{0}, v_{0}\right]$.

Proof. Since $F\left(u_{0}\right)$ is nonempty, there exists $u_{1} \in F\left(u_{0}\right)$ such that $u_{1} \geq u_{0}$. If $u_{1}=u_{0}$, then $u_{0}$ is a fixed point of $F$. So we can assume without loss of generality that $u_{1}>u_{0}$, therefore, $F\left(u_{0}\right) \leq^{s} F\left(u_{1}\right)$. So there is $u_{2} \in F\left(u_{1}\right)$ such that $u_{2} \geq u_{1}$, if $u_{2}=u_{1}$, then $u_{1}$ is a fixed point. Also, we can assume that $u_{2}>u_{1}$. In general, having defined $u_{n} \in F\left(u_{n-1}\right)$ such that $u_{n-1} \leq u_{n}$ if $u_{n}=u_{n-1}$, then $u_{n-1}$ is a fixed point of $F$. Without loss of generality, assume that $u_{n-1}<u_{n}$. Repeating this process, we can obtain an increasing sequence as follows:

$$
u_{0} \leq u_{1} \leq \cdots \leq u_{n} \leq \cdots \leq v_{0}
$$

Since $P$ is regular, there exists $u^{*}$ such that $u_{n} \rightarrow u^{*}$ as $n \rightarrow \infty$. On the other hand, from the facts $u_{n} \in F\left(u_{n-1}\right)$ and $F$ is upper semicontinuous, it follows that $u^{*} \in F\left(u^{*}\right)$. That is to say, $u^{*}$ is a fixed point of $F$.

Similarly, we can prove that there exists $v^{*}$ such that $v^{*} \in F\left(v^{*}\right)$.

In the following, we will show that $u^{*}, v^{*}$ are the maximal and minimal fixed points of $F$ on the order interval $\left[u_{0}, v_{0}\right]$, respectively.

Suppose that $u_{0} \leq \bar{x} \leq v_{0}$ with $\bar{x} \in F(\bar{x})$. Then $F\left(u_{0}\right) \leq^{s} F(\bar{x}) \leq^{s} F\left(v_{0}\right)$, so we obtain $u_{1} \leq \bar{x} \leq v_{1}$. Repeating this process, we have $F\left(u_{n-1}\right) \leq^{s} F(\bar{x}) \leq^{s} F\left(v_{n-1}\right)$, hence, $u_{n} \leq \bar{x} \leq$ $v_{n}$, passing the limit, $u^{*} \leq \bar{x} \leq v^{*}$. This completes the proof.

It is of interest to remark on the single-valued version of the Theorem 3.1.

Remark 3.2. (i) The conditions in Theorem 3.1 imply that $\left\{u_{0}\right\} \leq^{s} F\left(u_{0}\right), F\left(v_{0}\right) \leq^{s}\left\{v_{0}\right\}$.

(ii) If $u_{0} \in F\left(u_{0}\right)$, then $u_{0}$ is the minimal fixed point of $F$ in $D$. Otherwise, if $u_{i} \in$ $F\left(u_{i}\right)$ for certain $i \neq 0$, we can prove that $u_{i}$ is the minimal fixed point of $F$ in $D$. In fact, supposing that $\bar{u} \in F(\bar{u}), \bar{u} \in D, \bar{u} \neq u_{i}$, then $u_{0}<\bar{u}$. Similar to the proof of Theorem 3.1, we have $u_{i} \leq \bar{u}$. Similarly, if $v_{j} \in F\left(v_{j}\right)$ for certain $j$, then $v_{j}$ is the maximal fixed point of $F$ in $D$.

(iii) If $F$ is a singled-valued upper semicontinuous mapping, then it is continuous. 
3250 Multivalued maps in ordered Banach spaces

In addition, we have the following corollary.

Corollary 3.3 (see [5]). Let cone $P$ be regular and let $F: D \rightarrow X$ be a single-valued continuous, increasing mapping, assume that

$$
u_{0} \leq F\left(u_{0}\right), \quad F\left(v_{0}\right) \leq v_{0} .
$$

Then $F$ has maximal and minimal fixed points $x^{*}, x_{*}$ on $\left[u_{0}, v_{0}\right]$ and

$$
x^{*}=\lim _{n \rightarrow \infty} v_{n}, \quad x_{*}=\lim _{n \rightarrow \infty} u_{n}
$$

where $v_{n}=F\left(v_{n-1}\right), u_{n}=F\left(u_{n-1}\right)(n=1,2, \ldots)$ satisfy

$$
u_{0} \leq u_{1} \leq \cdots \leq u_{n} \leq v_{n} \leq \cdots \leq v_{1} \leq v_{0}
$$

Proof. Since $F: D \rightarrow X$ is a single-valued increasing mapping, and the facts $u_{0} \leq F\left(u_{0}\right)$, $F\left(v_{0}\right) \leq v_{0}$, we can obtain

$$
u_{0} \leq F\left(u_{0}\right) \leq F(x) \leq F\left(v_{0}\right) \leq v_{0}, \quad \forall x \in\left[u_{0}, v_{0}\right]
$$

Thus, $F: D \rightarrow D$. Note that $F: D \rightarrow X$ is continuous, we know that $F: D \rightarrow D$ is also upper semicontinuous. By Theorem 3.1, $F$ has maximal and minimal fixed points $x^{*}, x_{*}$ on $\left[u_{0}, v_{0}\right]$. From the proof of Theorem 3.1, (3.2) and (3.3) can be easily proved.

Theorem 3.4. Let $F: D \rightarrow 2^{D}$ be an upper semicontinuous, increasing, condensing mapping with nonempty value and let $P$ be a normal cone. Then $F$ has maximal and minimal fixed points on $\left[u_{0}, v_{0}\right]$.

Proof. From the proof of Theorem 3.1, we obtain

$$
\begin{aligned}
& u_{0} \leq u_{1} \leq \cdots \leq u_{n} \leq \cdots \leq v_{0}, \\
& v_{0} \geq v_{1} \geq \cdots \geq v_{n} \geq \cdots \geq u_{0},
\end{aligned}
$$

where $u_{n} \in F\left(u_{n-1}\right), v_{n} \in F\left(v_{n-1}\right)(n=1,2, \ldots), u_{n} \leq v_{n}$. Evidently, $\left\{u_{n}\right\},\left\{v_{n}\right\} \subset\left[u_{0}, v_{0}\right]$. Since $P$ is normal, then $\left\{u_{n}\right\},\left\{v_{n}\right\}$ are bounded sequences.

Suppose that $\alpha\left(\left\{u_{n}\right\}\right)>0$, it follows from $u_{n} \in F\left(u_{n-1}\right)$ that $\left\{u_{n}\right\} \subset F\left(\left\{u_{n}\right\}\right) \subset\left[u_{0}, v_{0}\right]$. Thus $\alpha\left(\left\{u_{n}\right\}\right) \leq \alpha\left(F\left(\left\{u_{n}\right\}\right)\right)$. Since $F$ is condensing, we have $\alpha\left(F\left(\left\{u_{n}\right\}\right)\right)<\alpha\left(\left\{u_{n}\right\}\right)$. This is a contradiction. So $\alpha\left(\left\{u_{n}\right\}\right)=0$, thus $\left\{u_{n}\right\}$ is relatively compact in $D$. Therefore, there exists a convergent subsequence $\left\{u_{n_{k}}\right\} \subset\left\{u_{n}\right\}$ and $u^{*} \in D$ such that $u_{n_{k}} \rightarrow u^{*}$ as $k \rightarrow \infty$.

Now we prove that $u_{n} \rightarrow u^{*}$. Suppose that this is not the case, then there exist a $\varepsilon_{0}>0$ and a subsequence $\left\{u_{n_{i}^{\prime}}\right\}$ of $\left\{u_{n}\right\}$ such that

$$
\left\|u_{n_{i}^{\prime}}-u^{*}\right\| \geq \varepsilon_{0} \quad(i=1,2, \ldots)
$$

By the relative compactness of $\left\{u_{n}\right\}$, we know that there exists a subsequence $\left\{u_{n_{i_{j}}^{\prime}}\right\}$ of $\left\{u_{n_{i}^{\prime}}\right\}$ such that $u_{n_{i_{j}}^{\prime}} \rightarrow \bar{u}(j \rightarrow \infty)$. Hence, forany given $k$, it follows from the increasing of $\left\{u_{n}\right\}$ that when $j$ is large enough, $u_{n_{k}} \leq u_{n_{i_{j}}^{\prime}}$. 
First letting $j \rightarrow \infty$ and then letting $k \rightarrow \infty$, we have $u^{*} \leq \bar{u}$.

Similarly, we can also prove that $\bar{u} \leq u^{*}$. Hence $u^{*}=\bar{u}$. This implies that when $j$ is large enough, we have

$$
\left\|u_{n_{i_{j}}^{\prime}}-u^{*}\right\|<\varepsilon_{0}
$$

This contradicts

$$
\left\|u_{n_{i}^{\prime}}-u^{*}\right\| \geq \varepsilon_{0} \quad(\forall i) .
$$

This completes the proof of $u_{n} \rightarrow u^{*}(n \rightarrow \infty)$.

Similarly, we can prove that there exists $v^{*}$ such that $v_{n} \rightarrow v^{*}(n \rightarrow \infty)$.

The remainder is same as Theorem 3.1. We omit it.

Corollary 3.5 (see [5]). Let $P$ be a normal cone and let $F: D \rightarrow X$ be a single-valued increasing, condensing mapping (condensing mapping $F$ possesses that $F$ is continuous). Assume that (3.2) holds. Then $F$ has maximal and minimal fixed points $x^{*}, x_{*}$ on $\left[u_{0}, v_{0}\right]$ such that (3.3), (3.4) hold.

Proof. Similar to the proof of Corollary 3.3, we have $F: D \rightarrow D$. Since $F: D \rightarrow X$ is a condensing mapping, we know that $F: D \rightarrow D$ is continuous. Thus, $F: D \rightarrow D$ is upper semicontinuous. So all the conditions of Theorem 3.4 are satisfied. The conclusion follows from Theorem 3.4.

Theorem 3.6. Let cone $P$ be strongly minimal and let $F: D \rightarrow 2^{D}$ be an increasing mapping with nonempty value. Then $F$ has fixed points $\bar{x}, \underline{x}$ on $\left[u_{0}, v_{0}\right]$, where

$$
\begin{aligned}
& \bar{x}=\sup \left\{x \mid u_{0} \leq x \leq v_{0}, u \geq x, \forall u \in F(x)\right\}, \\
& \underline{x}=\inf \left\{x \mid u_{0} \leq x \leq v_{0}, v \leq x, \forall v \in F(x)\right\} .
\end{aligned}
$$

Proof. Let $\Omega=\left\{x \mid u_{0} \leq x \leq v_{0}, u \geq x, \forall u \in F(x)\right\}$. Evidently, $\Omega$ is nonempty $\left(u_{0} \in \Omega\right)$ and $\Omega$ has an upper bound $v_{0}$. From $P$ being strongly minimal, we know that

$$
\bar{x}=\sup \Omega \text { exists, thus } u_{0} \leq \bar{x} \leq v_{0} .
$$

In the following, we prove that $\bar{x}$ is the fixed point of $F$ on $\left[u_{0}, v_{0}\right]$.

For $x \in \Omega, u_{0} \leq x \leq \bar{x} \leq v_{0}$ holds. So we obtain

$$
\left\{u_{0}\right\} \leq^{s} F(x) \leq^{s} F(\bar{x}) \leq^{s}\left\{v_{0}\right\} .
$$

Since $\{x\} \leq^{s} F(x)$, then $\{x\} \leq^{s} F(\bar{x})$, which implies that $u \geq x$ for all $u \in F(\bar{x})$. That is to say, for all $u \in F(\bar{x})$ is the upper bound of $\Omega$. Therefore, $\bar{x} \leq u$ for all $u \in F(\bar{x})$. Hence, $F(\bar{x}) \leq^{s} F(u)$ for all $u \in F(\bar{x})$. So $\{u\} \leq^{s} F(u)$ for $\forall u \in F(\bar{x})$. By the definition of $\Omega$, we have $u \in \Omega$, thus $F(\bar{x}) \subset \Omega$. It follows that $F(\bar{x}) \leq^{s}\{\bar{x}\}$. So $u \leq \bar{x}$ for all $u \in F(\bar{x})$. Consequently, $\bar{x}=u$, namely, $F(\bar{x})=\{\bar{x}\}$ (a singleton). This implies that $\bar{x}$ is the fixed point of $F$ on $\left[u_{0}, v_{0}\right]$. 
3252 Multivalued maps in ordered Banach spaces

Similarly, we can prove that

$$
\underline{x}=\inf \left\{x \mid u_{0} \leq x \leq v_{0}, v \leq x, \forall v \in F(x)\right\}
$$

is the fixed point of $F$ on $\left[u_{0}, v_{0}\right]$.

Remark 3.7. Here we remove the assumption that $F$ is upper semicontinuous. If $F$ is a single-valued mapping, then

$$
\begin{aligned}
& \bar{x}=\sup \left\{x \mid u_{0} \leq x \leq v_{0}, F(x) \geq x\right\} \\
& \underline{x}=\inf \left\{x \mid u_{0} \leq x \leq v_{0}, F(x) \leq x\right\}
\end{aligned}
$$

are the maximal and minimal fixed points of $F$ on $\left[u_{0}, v_{0}\right]$. In fact, suppose that there is $x^{*} \in\left[u_{0}, v_{0}\right]$ such that $F\left(x^{*}\right)=x^{*}$, then we have $x^{*} \in\left\{x \mid u_{0} \leq x \leq v_{0}, F(x) \geq x\right\}$ and $x^{*} \in\left\{x \mid u_{0} \leq x \leq v_{0}, F(x) \leq x\right\}$. Thus, $\underline{x} \leq x^{*} \leq \bar{x}$. This shows the following corollary.

Corollary 3.8 (see [1]). Let cone $P$ be strongly minimal and let $F: D \rightarrow X$ be a singlevalued increasing mapping. In addition, (3.2) holds. Then $F$ has maximal and minimal fixed points on $\left[u_{0}, v_{0}\right]$.

\section{Necessity and sufficiency}

In this section, we present a necessary and sufficient condition for the existence of the fixed point to multivalued maps which satisfy some conditions. In addition, we can obtain the uniqueness result under relatively strong conditions. We also do not assume that the mapping is upper semicontinuous.

Theorem 4.1. Let cone $P$ be normal and let $F: P_{h} \rightarrow 2^{P_{h}}$ be an increasing mapping with nonempty value. In addition, there exist $\mu \in(0,1)$ such that

$$
F(t x) \geq^{s} t^{\mu} F(x), \quad \text { for } t \in(0,1), \forall x \in P_{h} .
$$

Then a necessary and sufficient condition for $F$ to have a fixed point in $P_{h}$ is that the following holds. There exist $u_{0}, v_{0} \in P_{h}$ with $u_{0}<v_{0}$ such that

$$
\left\{u_{0}\right\} \leq^{s} F\left(u_{0}\right), \quad F\left(v_{0}\right) \leq^{s}\left\{v_{0}\right\} .
$$

Proof. (1) Sufficiency. From the proof of Theorem 3.1, we obtain

$$
\begin{aligned}
& u_{0} \leq u_{1} \leq \cdots \leq u_{n} \leq \cdots \leq v_{0}, \\
& v_{0} \geq v_{1} \geq \cdots \geq v_{n} \geq \cdots \geq u_{0},
\end{aligned}
$$

where $u_{n} \in F\left(u_{n-1}\right), v_{n} \in F\left(v_{n-1}\right)(n=1,2, \ldots), u_{n} \leq v_{n}$. The fact that $u_{0}, v_{0} \in P_{h}, u_{0}<$ $v_{0}$ shows that there is $\lambda_{0} \in(0,1)$ such that $u_{0} \geq \lambda_{0} v_{0}$, then we have $\lambda_{0}{ }^{\mu} F\left(v_{0}\right) \leq^{s} F\left(u_{0}\right)$. This implies that $u_{1} \geq \lambda_{0}{ }^{\mu} v_{1}$, then $\lambda_{0}{ }^{\mu^{2}} F\left(v_{1}\right) \leq^{s} F\left(u_{1}\right)$, and so $u_{2} \geq \lambda_{0}{ }^{\mu^{2}} v_{2}$. Repeating this process, we can obtain $u_{n} \geq \lambda_{0}{ }^{\mu^{n}} v_{n}$. Therefore,

$$
0 \leq v_{n}-u_{n} \leq v_{n}-\lambda_{0}{ }^{\mu^{n}} v_{n}=\left(1-\lambda_{0}{ }^{\mu^{n}}\right) v_{n}<\left(1-\lambda_{0}{ }^{\mu^{n}}\right) v_{0} .
$$


By the normality of cone $P$, we have

$$
\left\|v_{n}-u_{n}\right\| \leq N\left(1-\lambda_{0}{ }^{\mu^{n}}\right)\left\|v_{0}\right\| \longrightarrow 0 \quad(n \longrightarrow \infty),
$$

here $N$ is the normal constant. Further,

$$
\begin{gathered}
0 \leq u_{n+p}-u_{n} \leq v_{n}-u_{n}, \\
0 \leq v_{n}-v_{n+p} \leq v_{n}-u_{n} \quad(p \in N) .
\end{gathered}
$$

Thus,

$$
\begin{array}{ccc}
\left\|u_{n+p}-u_{n}\right\| \leq N\left\|v_{n}-u_{n}\right\| \longrightarrow 0 & & (n \longrightarrow \infty), \\
\left\|v_{n}-v_{n+p}\right\| \leq N\left\|v_{n}-u_{n}\right\| \longrightarrow 0 & & (n \longrightarrow \infty) .
\end{array}
$$

So we can claim that $\left\{u_{n}\right\},\left\{v_{n}\right\}$ are Cauchy sequences. Then there exists $u^{*}$ such that $u_{n} \rightarrow u^{*}$ as $n \rightarrow \infty$ and $v_{n} \rightarrow u^{*}$ as $n \rightarrow \infty$. It follows that $u_{n}<u^{*}<v_{n}$. Thus, $F\left(u_{n}\right) \leq^{s}$ $F\left(u^{*}\right) \leq^{s} F\left(v_{n}\right)$. Since $u_{n+1} \in F\left(u_{n}\right), v_{n+1} \in F\left(v_{n}\right)$, we obtain

$$
u_{n+1} \leq u \leq v_{n+1}, \quad \forall u \in F\left(u^{*}\right),
$$

passing the limit in (4.8), $u=u^{*}$, namely, $F\left(u^{*}\right)=\left\{u^{*}\right\}$. This implies that $u^{*}$ is a fixed point of $F$.

(2) Necessity. Let $x_{0}$ be the fixed point of $F$, then $t^{\mu} F\left(x_{0}\right) \leq^{s} F\left(t x_{0}\right)$. Take $t_{0}$ sufficiently small such that $t_{0}{ }^{\mu}>t_{0}$, then

$$
\left\{t_{0} u\right\} \leq^{s}\left\{t_{0}{ }^{\mu} u\right\} \leq^{s} F\left(t_{0} x_{0}\right), \quad \forall u \in F\left(x_{0}\right),
$$

which implies that

$$
\left\{t_{0} u \mid \forall u \in F\left(x_{0}\right)\right\} \leq^{s} F\left(t_{0} x_{0}\right),
$$

that is, $t_{0} F\left(x_{0}\right) \leq{ }^{s} F\left(t_{0} x_{0}\right)$. It follows from $t^{\mu} F\left(x_{0}\right) \leq{ }^{s} F\left(t x_{0}\right)$ that

$$
F\left(x_{0}\right)=F\left(t \cdot \frac{1}{t} x_{0}\right) \geq^{s} t^{\mu} F\left(\frac{1}{t} x_{0}\right) .
$$

So we have

$$
F\left(\frac{1}{t} x_{0}\right) \leq^{s} \frac{1}{t^{\mu}} F\left(x_{0}\right)
$$

and we can choose $t_{1}$ sufficiently small satisfying $1 / t_{1}{ }^{\mu}<1 / t_{1}$, then

$$
F\left(\frac{1}{t_{1}} x_{0}\right) \leq^{s}\left\{\frac{1}{t_{1}^{\mu}} u\right\} \leq^{s}\left\{\frac{1}{t_{1}} u\right\}, \quad \forall u \in F\left(x_{0}\right) .
$$

That is,

$$
F\left(\frac{1}{t_{1}} x_{0}\right) \leq^{s}\left\{\frac{1}{t_{1}} u \mid \forall u \in F\left(x_{0}\right)\right\}=\frac{1}{t_{1}} F\left(x_{0}\right) .
$$


Now we write $\bar{t}=\min \left\{t_{0}, t_{1}\right\}<1, u_{0}=\bar{t} x_{0}, v_{0}=(1 / \bar{t}) x_{0}$. Then

$$
u_{0}<v_{0}, \quad\left\{u_{0}\right\} \leq^{s} F\left(u_{0}\right), \quad F\left(v_{0}\right) \leq^{s}\left\{v_{0}\right\} .
$$

Taking $\lambda_{0} \in\left(0, \bar{t}^{2}\right)$, we have $u_{0} \geq \lambda_{0} v_{0}$. The proof is complete.

The uniqueness of the positive fixed point appears somewhat difficult to study. In the following, we give a result under relatively strong conditions.

Theorem 4.2. Let cone $P$ be normal and let $F: P_{h} \rightarrow 2^{D}$ be a strictly increasing mapping (i.e., $x<y$ implies that $F(x)<{ }^{s} F(y)$ ) with nonempty value. In addition, assume that

(i) $u_{0}, v_{0} \in P_{h}$ and $u_{0} \notin F\left(u_{0}\right), v_{0} \notin F\left(v_{0}\right)$;

(ii) there exists $\mu \in(0,1)$ such that $F(t x) \geq^{s} t^{\mu} F(x)$ for $t \in(0,1)$, for all $x \in P$.

Then $F$ has a unique fixed point in $D$.

Proof. From (i), we have

$$
u_{0}<u_{1}<\cdots<u_{n}<\cdots<v_{n}<\cdots<v_{1}<v_{0},
$$

where $u_{n} \in F\left(u_{n-1}\right), v_{n} \in F\left(v_{n-1}\right)(n=1,2, \ldots)$.

From the sufficiency of Theorem 4.1 , we know that there exists $u^{*} \in D$ such that $u_{n} \rightarrow$ $u^{*}, v_{n} \rightarrow u^{*}, u^{*} \in F\left(u^{*}\right)$.

In the following, we prove that $u^{*}$ is the unique fixed point of $F$ in $D$. In fact, suppose that $\bar{u}$ is the fixed point of $F$ with $\bar{u} \neq u^{*}$, then $u_{0}<\bar{u}<v_{0}$. So $F\left(u_{0}\right)<{ }^{s} F(\bar{u})<{ }^{s} F\left(v_{0}\right)$, thus $u_{1}<\bar{u}<v_{1}$. Repeating this process, we have

$$
u_{n}<\bar{u}<v_{n}
$$

passing the limit in (4.17), $\bar{u}=u^{*}$. This is a contradiction. This completes the proof.

From a theoretical viewpoint, it is of interest to discuss the single-valued version of Theorems 4.1 and 4.2 , then in that case, the operator is called $\mu$-convex, some results of this type can be found in $[4,14]$.

\section{Applications}

It is well known that concave and convex operators defined on a cone in a Banach space play an important role in theory of positive operators (see e.g., [7, Chapter 6]). In [14], Potter introduced the definitions of $\alpha$-concave operators and $\alpha$-convex operators, he shows that for $\alpha \geq 0$, increasing $\alpha$-concave and decreasing $(-\alpha)$-convex mappings have contraction ratios less than or equal to $\alpha$ and give the existence of solutions to the nonlinear eigenvalue problem $A x=\lambda x$. The method is based upon Hilbert's projective metric (see [2] for details). In [15], the author improves the corresponding results presented in [14] by using contraction mapping theorem. In [4], Guo widens the conditions and removes the hypotheses of continuation for operators, and then extends the results of fixed points, eigenvectors for $\alpha$-concave $(-\alpha$-convex) operators. However, they restrict their attention to $0<|\alpha| \leq 1$, while for the remaining cases $\alpha>1$ and $\alpha<-1$, the research proceeds slowly and appears difficult because Hilbert's projective metric is useless for these cases. Up to now, pleasant results are seldom obtained. 
The aim of this section is to obtain the existence and uniqueness of positive fixed points for $\alpha$-convex $(\alpha>1)$ operators by using Theorem 4.1 .

Theorem 5.1. Let E be a real Banach space and let $P$ be a normal cone in E. Let $A: P_{h} \rightarrow P_{h}$ be an increasing $\alpha$-convex operator $(\alpha>1)$, that is, $A(t x) \leq t^{\alpha} A x$ for all $x \in P_{h}$ and $t \in$ $(0,1]$. Assume that there exists a nonempty, totally ordered set $S \subset P_{h}$ such that

(i) $\lambda S \subset S(\lambda \in(0,1)), A S=S$;

(ii) $A v_{0} \geq v_{0}$ for certain $v_{0} \in S$.

Then $A$ has exactly one positive fixed point in $S$.

Proof. Now we divide the proof into several steps.

Step 1. Consider the operator $A: S \rightarrow S$. For all $y \in S$, set $A^{-1} y=\{x \in S: A x=y\}$. Then $A^{-1}: S \rightarrow 2^{S}$ is a set-valued mapping. Consequently, we have the following conclusions.

(a) $A^{-1}$ is increasing in the sense of set-valued mappings.

In fact, if $y_{1}>y_{2}$ for all $y_{1}, y_{2} \in S$, then $A^{-1} y_{1} \geq^{s} A^{-1} y_{2}$. Suppose that this is not true, then we have $x_{1}<x_{2}$ for all $x_{1} \in A^{-1} y_{1}, x_{2} \in A^{-1} y_{2}$. Using the monotonicity of $A$, we have $A x_{1} \leq A x_{2}$, that is to say, $y_{1} \leq y_{2}$, this is a contradiction.

(b) $A^{-1}(s y) \geq{ }^{s} s^{1 / \alpha} A^{-1} y$ for $y \in S, s \in(0,1)$.

For $x \in A^{-1} y$, then $A x=y$ and $A(t x) \leq t^{\alpha} A x$, for $t \in(0,1)$. Let $s=t^{\alpha}$, then we have $A\left(s^{1 / \alpha} x\right) \leq s A x$, and (a) implies that $A^{-1}\left(A\left(s^{1 / \alpha} x\right)\right) \leq^{s} A^{-1}(s A x)$. Thus

$$
\left\{s^{1 / \alpha} x\right\} \leq^{s} A^{-1}(s y), \quad \forall x \in A^{-1} y .
$$

By the arbitrariness of $x$, one obtains that

$$
\left\{s^{1 / \alpha} x \mid x \in A^{-1} y\right\} \leq^{s} A^{-1}(s y)
$$

namely, $s^{1 / \alpha} A^{-1} y \leq^{s} A^{-1}(s y)$.

Step 2. For $v_{0} \in S, t \in(0,1)$, we have $A\left(t v_{0}\right) \leq t^{\alpha} A v_{0}$. Since $A v_{0} \in S \subset P_{h}$, there exist $\lambda, \mu>$ 0 such that $\lambda v_{0} \leq A v_{0} \leq \mu v_{0}$, so $A\left(t v_{0}\right) \leq t^{\alpha} A v_{0} \leq \mu t^{\alpha} v_{0}$. So we can choose $t_{1}$ sufficiently small satisfying $\mu t^{\alpha}<t$, then $A\left(t_{1} v_{0}\right) \leq t_{1} v_{0}, t_{1} \in(0,1)$.

Now we write $u_{0}=t_{1} v_{0}$, then $A u_{0} \leq u_{0}$ and $u_{0}<v_{0}$. Taking $\lambda_{0}=t_{1}{ }^{2}$, then $\lambda_{0} \in(0,1)$, $u_{0}=t_{1} v_{0} \geq t_{1}^{2} v_{0}=\lambda_{0} v_{0}$.

Step 3. By Step 2 and (ii), we know that

$$
u_{0}, v_{0} \in S, \quad u_{0}<v_{0}, \quad u_{0} \geq \lambda_{0} v_{0}, \quad A u_{0} \leq u_{0}, \quad A v_{0} \geq v_{0}
$$

Evidently, $A^{-1} u_{0} \geq^{s}\left\{u_{0}\right\}, A^{-1} v_{0} \leq^{s}\left\{v_{0}\right\}$. An application of the sufficiency of Theorem 4.1 shows that $A^{-1}$ has a fixed point, write $u^{*}$, which implies that $A u^{*}=u^{*}$. That is to say, $u^{*}$ is the positive fixed point of $A$.

Step 4. In the following, we prove that $u^{*}$ is the unique fixed point of $A$ in $S$. 
In fact, suppose that $\bar{u}$ is the fixed point of $A$ with $\bar{u} \neq u^{*}$. Evidently, $\bar{u}, u^{*}>\theta$. Since $S$ is a totally ordered set, we assume without loss of generality that $\bar{u}>u^{*}$, write $x_{1}=u^{*}$, $x_{2}=\bar{u}$, then $x_{2}>x_{1}$. The fact that $x_{1}, x_{2} \in P_{h}$ shows that there exists $\mu_{0}>0$ such that $x_{1} \geq \mu_{0} x_{2}$. Obviously, $\mu_{0}<1$.

If $x_{1}=\mu_{0} x_{2}$, then $A x_{1}=A\left(\mu_{0} x_{2}\right) \leq \mu_{0}{ }^{\alpha} A x_{2}$, that is, $x_{1} \leq \mu_{0}{ }^{\alpha} x_{2}<\mu_{0} x_{2}$, this is a contradiction. Thus $x_{1}>\mu_{0} x_{2}$, so $A^{-1} x_{1} \geq^{s} A^{-1}\left(\mu_{0} x_{2}\right) \geq^{s} \mu_{0}{ }^{1 / \alpha} A^{-1} x_{2}$, this shows that $x_{1} \geq$ $\mu_{0}^{1 / \alpha} x_{2}$. If $x_{1}=\mu_{0}^{1 / \alpha} x_{2}$, then $A\left(x_{1}\right)=A\left(\mu_{0}^{1 / \alpha} x_{2}\right) \leq \mu_{0} x_{2}$, this is a contradiction. Hence $x_{1}>\mu_{0}{ }^{1 / \alpha} x_{2}$, repeating this process, we obtain $x_{1}>\mu_{0}{ }^{1 / \alpha^{n}} x_{2}$. Consequently,

$$
\theta<x_{2}-x_{1}<x_{2}-\mu_{0}^{1 / \alpha^{n}} x_{2}=\left(1-\mu_{0}^{1 / \alpha^{n}}\right) x_{2}
$$

by the normality of cone $P$, we have

$$
\left\|x_{2}-x_{1}\right\| \leq N\left(1-\mu_{0}^{1 / \alpha^{n}}\right)\left\|x_{2}\right\| \longrightarrow 0 \quad(n \longrightarrow \infty),
$$

thus, $x_{1}=x_{2}$, this is a contradiction. This completes the proof.

Corollary 5.2. Let $E$ be a real Banach space and let $P$ be a normal cone in E. Let $A$ : $P_{h} \rightarrow P_{h}$ be an increasing $\alpha$-convex operator $(\alpha>1)$, that is, $A(t x) \leq t^{\alpha} A x$ for all $x \in P_{h}$ and $t \in(0,1]$. Assume that there exists a nonempty, totally ordered set $S \subset P_{h}$ such that $\lambda S \subset S(\lambda>0), A S=S$. Then $A$ has exactly one positive fixed point in $S$.

Proof. From the proof of Theorem 5.1, we only have to prove that there exists $v_{0} \in S$ such that $A v_{0} \geq v_{0}$.

For all $x_{0} \in S, s>1$, we have $A\left(s x_{0}\right) \geq s^{\alpha} A x_{0}$. Since $A x_{0} \in S \subset P_{h}$, there exist $\mu, \lambda>0$ such that $\lambda x_{0} \leq A x_{0} \leq \mu x_{0}$, thus $A\left(s x_{0}\right) \geq s^{\alpha} \lambda x_{0}$. So we can choose $s_{0}$ large enough such that $s_{0}^{\alpha} \lambda \geq s_{0}$. Hence, $A\left(s_{0} x_{0}\right) \geq s_{0} x_{0}$. Now we write $v_{0}=s_{0} x_{0}$, then $A v_{0} \geq v_{0}$. The proof is complete.

Remark 5.3. (i) Under the hypotheses of this section, the tool-Hilbert's projective metric-used in those papers $[2,3,14]$ cannot be used.

(ii) By using the properties of inverse mapping, (set-valued mapping) we give the similar results to $\alpha$-concave operators $(0<\alpha<1)$. So our results compliment the theory of concave and convex operators and the method is new and different from previous ones.

Example 5.4. Theorem 5.1 or Corollary 5.2 can be used to discuss the solution of the following special integral equation:

$$
x(t)=\int_{0}^{1} k(t, s) x^{\alpha}(s) d s, \quad \alpha>1
$$

Suppose that $k(t, s)=h(t) f(s)$, and $h, f$ are nonnegative continuous functions with $f(t)>$ $0, h(t)>0$, for $t \in[0,1]$. Then, (5.6) has exactly one positive continuous solution.

Proof. First, we have some notation. Put $X=C[0,1]$ (the space of continuous functions defined on $[0,1]$ endowed with supremum norm). Let $P$ be the cone of nonnegative 
functions in $X$ and so $P$ is normal, $\stackrel{\circ}{P}$ is the set of positive functions in $X$. Noting that $P$ is a closed solid, the norm is monotonic. Consider the integral operator $A: \stackrel{\circ}{P} \rightarrow X$ defined by

$$
A x(t)=\int_{0}^{1} k(t, s) x^{\alpha}(s) d s, \quad x \in \stackrel{\circ}{P} .
$$

Therefore,

$$
A x(t)=h(t) \int_{0}^{1} f(s) x^{\alpha}(s) d s=a h(t)
$$

where $a=\int_{0}^{1} f(s) x^{\alpha}(s) d s=x^{\alpha}(\xi) \int_{0}^{1} f(s) d s$, for certain $\xi \in(0,1)$. Evidently, $a>0, a h \in \stackrel{\circ}{P}$, so $A: \stackrel{\circ}{P} \rightarrow \stackrel{\circ}{P}$. Let $S=\left\{x: x(t)=a h(t), a \in \mathbb{R}^{+}\right\}$, then we have $S \subset \stackrel{\circ}{P}$ and $S$ is a totally ordered set with $\lambda S \subset S(\lambda>0)$. In the following, we prove that $A S=S$. For all $y \in S, y=$ $a h$, there is $x=(a / H)^{1 / \alpha} h \in S$ such that $A x=y$, where $H=\int_{0}^{1} f(s) h^{\alpha}(s) d s$. Thus, $A S=S$. Since $A$ is increasing in $\stackrel{\circ}{P}$, Theorem 5.1 implies that $A$ has exactly one fixed point $x^{*}$ in $S$. Further, $x^{*}=H^{1 / 1-\alpha} h$. In fact, let $x^{*}=a_{0} h$, then $A x^{*}=A\left(a_{0} h\right)=a_{0}{ }^{\alpha} A h=a_{0}{ }^{\alpha} H h=a_{0} h$. So we obtain $a_{0}=H^{1 / 1-\alpha}$, thus (5.6) has one positive solution $x^{*}(t)=H^{1 / 1-\alpha} h(t)$.

Remark 5.5. For (5.6), we can also use the following lemma generalized from $[2,3]$ to prove the results.

Lemma 5.6. Let the norm in Banach space $X$ be monotonic on cone $P$, let $A: \stackrel{\circ}{P} \rightarrow \stackrel{\circ}{P}$ be positive homogeneous of degree $p, 0<|p|<1$ (i.e., $A(t x)=t^{p} A x, \forall x \in \stackrel{\circ}{P}, t>0$ ). In addition, $A$ is increasing $(0<p<1)$ or decreasing $(-1<p<0)$. Then $A$ has exactly one positive fixed point in $\stackrel{\circ}{P}$.

Proof of (5.6). As in the proof above, $A: S \rightarrow S, A S=S$. For $x_{1}, x_{2} \in S$ with $x_{1} \neq x_{2}$, then $A x_{1} \neq A x_{2}$. In fact, let $x_{1}=a_{1} h, x_{2}=a_{2} h, a_{1} \neq a_{2}, a_{1}, a_{2}>0$. Then $A x_{1}=A\left(a_{1} h\right)=a_{1}^{\alpha} A h$, and $A x_{2}=A\left(a_{2} h\right)=a_{2}{ }^{\alpha} A h$, this together with $a_{1}{ }^{\alpha} \neq a_{2}{ }^{\alpha}$ implies the conclusion. So $A$ is one-to-one mapping, consequently, $A^{-1}: S \rightarrow S$ exists, further,

$$
A^{-1}(t x)=t^{1 / \alpha} A^{-1} x, \quad t \in(0,1) .
$$

Since $A$ is strictly increasing, we obtain that $A^{-1}$ is also increasing. Otherwise, for $y_{1}, y_{2} \in$ $S, y_{1} \leq y_{2}$, we have $A^{-1} y_{1}>A^{-1} y_{2}$, thus, $A A^{-1} y_{1}>A A^{-1} y_{2}$, that is, $y_{1}>y_{2}$. This is a contradiction. An application of Lemma 5.6 implies that $A^{-1}$ has exactly one positive fixed point in $S$. Since $A h=H h, A^{-1}(\lambda x)=\lambda^{1 / \alpha} A^{-1} x$. We have $A^{-1} h=H^{-1 / \alpha} h$. Let $u_{0}=h$, $u_{n}=A^{-1} u_{n-1}(n=1,2, \ldots)$. Then

$$
\begin{gathered}
u_{1}=A^{-1} u_{0}=A^{-1} h=H^{-1 / \alpha} h, \\
u_{2}=A^{-1} u_{1}=A^{-1}\left(H^{-1 / \alpha} h\right)=\left(H^{-1 / \alpha}\right)^{(1+1 / \alpha)} h, \\
\vdots \\
u_{n+1}=A^{-1} u_{n}=\left(H^{-1 / \alpha}\right)^{\left(1+1 / \alpha+\cdots+1 / \alpha^{n}\right)} h=H^{\left(1-1 / \alpha^{n}\right) /(1-\alpha)} h .
\end{gathered}
$$


Thus, we have

$$
\left\|u_{n+1}-H^{1 / 1-\alpha} h\right\|=\left|H^{\left(1-1 / \alpha^{n}\right) /(1-\alpha)}-H^{1 / 1-\alpha}\right|\|h\| \longrightarrow 0 \quad(n \longrightarrow \infty) ;
$$

consequently, $u_{n} \rightarrow H^{1 / 1-\alpha} h$. In addition,

$$
A^{-1}\left(H^{1 / 1-\alpha} h\right)=\left(H^{1 / 1-\alpha}\right)^{1 / \alpha} H^{-1 / \alpha} h=H^{1 / 1-\alpha} h .
$$

which implies that $H^{1 / 1-\alpha} h$ is the fixed point of $A^{-1}$. Hence, $A$ has exactly one positive fixed point $H^{1 / 1-\alpha} h$ in $S$.

Remark 5.7. For the uniqueness of the solution of (5.6), we can also prove it by using the following method.

Proof. Let $x_{1}, x_{2}$ be the solutions of (5.6). Noting that $x_{1}=a_{1} h, x_{2}=a_{2} h$, then $x_{1}=a_{1} / a_{2}$, $x_{2}=a x_{2}$, where $a=a_{1} / a_{2}$, evidently, $0<a<\infty$.

When $a>1$, we have $A x_{1}=A\left(a x_{2}\right)=a^{\alpha} A x_{2}=a^{\alpha} x_{2}>a x_{2}$, that is, $x_{1}>a x_{2}$. This is a contradiction. When $a<1$, we have $A x_{1}=A\left(a x_{2}\right)=a^{\alpha} A x_{2}=a^{\alpha} x_{2}<a x_{2}$, that is, $x_{1}<a x_{2}$. This is a contradiction. So $a=1$, and we obtain $x_{1}=x_{2}$. This completes the proof.

\section{Acknowledgment}

This research is supported by the fund of National Natural Science of China (10371068).

\section{References}

[1] H. Amann, On the number of solutions of nonlinear equations in ordered Banach spaces, J. Functional Analysis 11 (1972), no. 3, 346-384.

[2] P. J. Bushell, Hilbert's metric and positive contraction mappings in a Banach space, Arch. Rational Mech. Anal. 52 (1973), no. 4, 330-338.

[3] - On a class of Volterra and Fredholm non-linear integral equations, Math. Proc. Cambridge Philos. Soc. 79 (1976), no. 2, 329-335.

[4] D. J. Guo, Fixed points and eigenelements of a class of concave and convex operators, Kexue Tongbao (Chinese) 30 (1985), no. 15, 1132-1135 (Chinese).

[5] Nonlinear Functional Analysis, Shandong Science and Technology Press, Jinan, 1985.

[6] D. J. Guo and V. Lakshmikantham, Nonlinear Problems in Abstract Cones, Notes and Reports in Mathematics in Science and Engineering, vol. 5, Academic Press, Massachusetts, 1988.

[7] M. A. Krasnoselskii, Positive Solutions of Operator Equations, Noordhoff, Groningen, 1964.

[8] M. A. Krasnoselskii, J. A. Lifshits, and A. V. Sobolev, Positive Linear Systems. The Method of Positive Operators, Sigma Series in Applied Mathematics, vol. 5, Heldermann, Berlin, 1989.

[9] K. Kuratowski, Sur les espaces complets, Fund. Math. 15 (1930), 301-309 (French).

[10] V. Lakshmikantham and S. Leela, Nonlinear Differential Equations in Abstract Spaces, International Series in Nonlinear Mathematics: Theory, Methods and Applications, vol. 2, Pergamon Press, Oxford, 1981.

[11] Z. Liang, L. Zhang, and S. Li, Fixed point theorems for a class of mixed monotone operators, Z. Anal. Anwendungen 22 (2003), no. 3, 529-542.

[12] R. H. Martin Jr., Nonlinear Operators and Differential Equations in Banach Spaces, Pure and Applied Mathematics, John Wiley \& Sons, New York, 1976.

[13] D. O'Regan, Existence and approximation of fixed points for multivalued maps, Appl. Math. Lett. 12 (1999), no. 6, 37-43. 
[14] A. J. B. Potter, Applications of Hilbert's projective metric to certain classes of non-homogeneous operators, Quart. J. Math. Oxford Ser. (2) 28 (1977), no. 109, 93-99.

[15] W. Weixun, Conditions for contraction of mappings and Banach type fixed point theorem, Acta Math. Sci. 27 (1984), 35-52 (Chinese).

Zhai Chengbo: Department of Mathematics, Shanxi University, Taiyuan 030006, Shanxi, China E-mail address: cbzhai215@sohu.com

Yang Chen: Department of Mathematics, Shanxi University, Taiyuan 030006, Shanxi, China E-mail address: cbzhai@sina.com.cn 


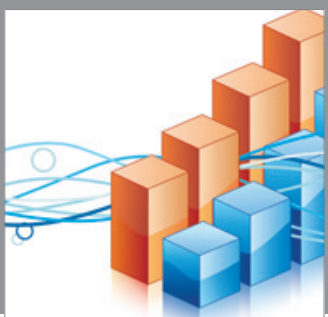

Advances in

Operations Research

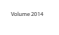

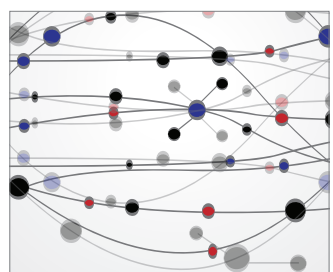

\section{The Scientific} World Journal
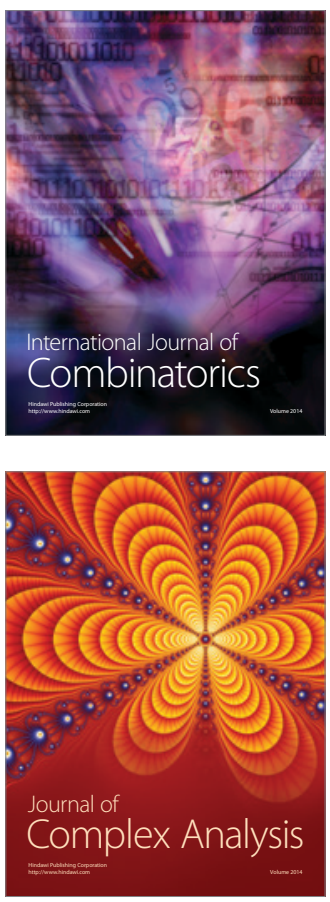

International Journal of

Mathematics and

Mathematical

Sciences
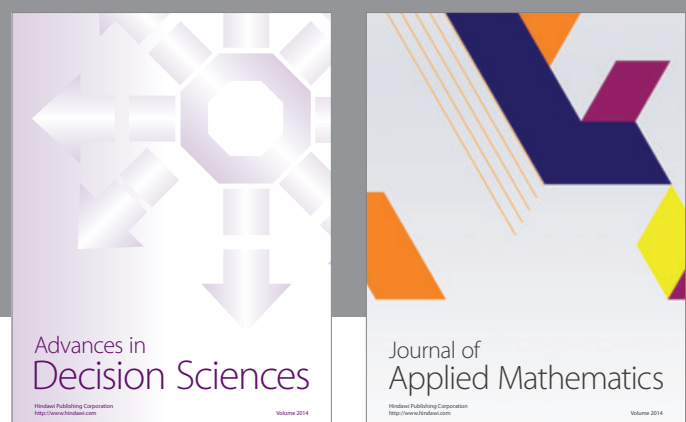

Journal of

Applied Mathematics
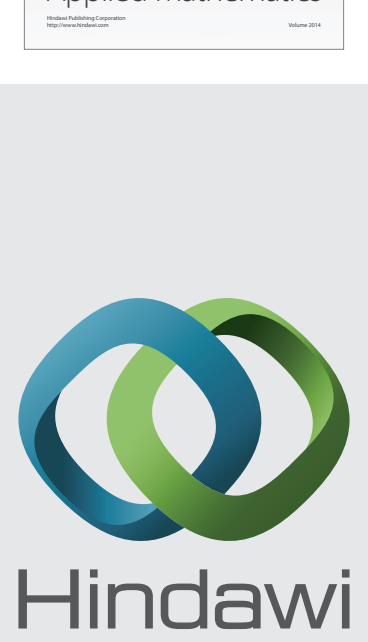

Submit your manuscripts at http://www.hindawi.com
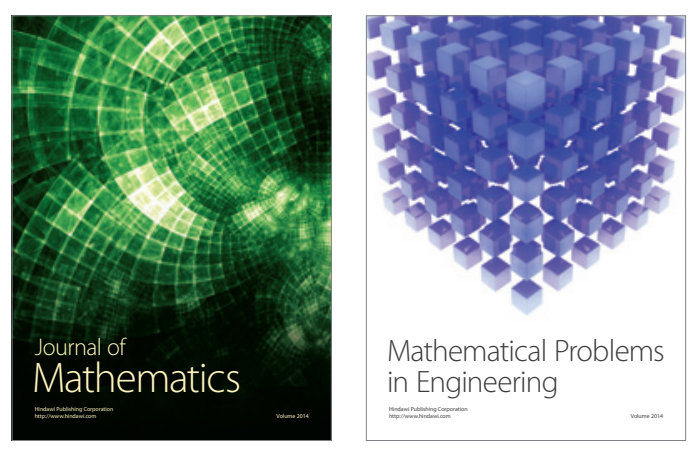

Mathematical Problems in Engineering
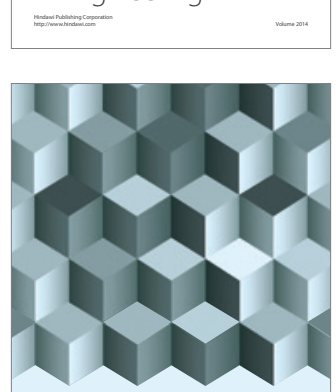

Journal of

Function Spaces
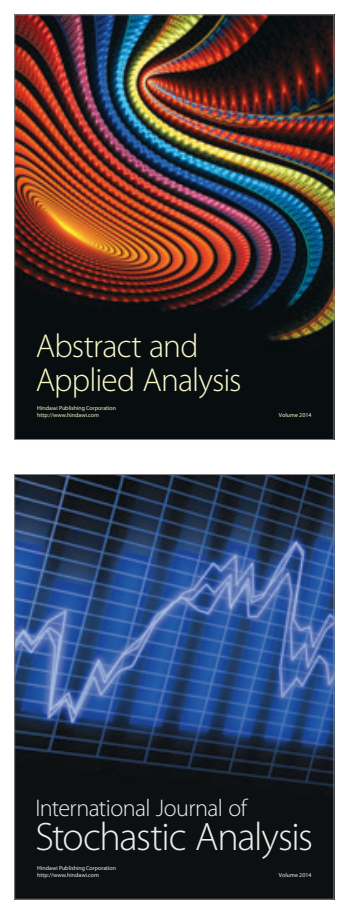

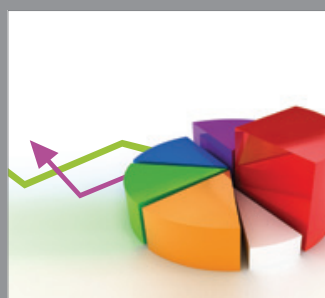

ournal of

Probability and Statistics

Promensencen
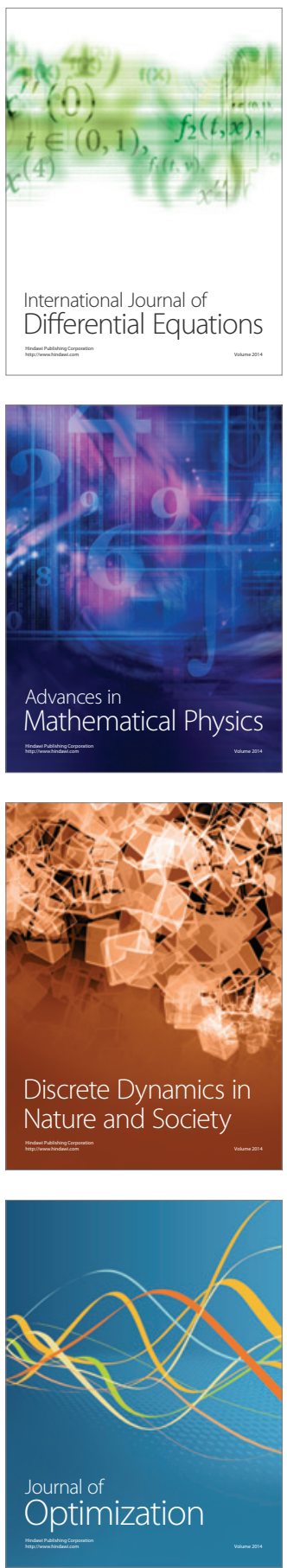